\title{
Using Genersys to model electricity generation expansion
}

\author{
$\underline{\text { M. James }}^{\text {a }}$, G. Grozev ${ }^{\text {a }}$, J. Page ${ }^{\text {a }}$, and K-K. Yum ${ }^{\text {a }}$ \\ ${ }^{a}$ CSIRO Ecosystem Sciences, 37 Graham Road, Highett, Victoria 3190 \\ Email: melissa.james@csiro.au
}

\begin{abstract}
In an environment of increasing complexity, with new electricity generation technologies, climate change, carbon pricing, and fluctuating fuel prices, electricity generation expansion planners need tools which facilitate the integration and synthesis of a wide range of complex and often uncertain information to support decision making for investment.
\end{abstract}

This paper presents a capacity expansion algorithm integrated in Genersys - an agent-based simulation model for electricity and gas markets. The algorithm evaluates different electricity generation technologies for investment in new generating plants in different regions of the market as a response to growing electricity demand, unserved energy and/or high wholesale prices of electricity. It considers capacity factors associated with peak, intermediate and base load generation, technology and fuel costs, carbon price, and distribution of recent electricity prices. As a fully integrated simulation algorithm within Genersys, it can be used to assess complex investment scenarios defined by demand models, bidding behaviour of generators, non-scheduled renewable generation, outage models and all other components modelled by this simulation tool. The algorithm creates modular expansions - a single generating unit with predefined generation capacity of the best ranked generation technology in a region will be invested in if the investment criteria are satisfied. Generating plants newly created by the capacity expansion algorithm begin to participate in the market, producing and selling electricity. They will have immediate impact on the balance between demand and supply and ultimately will influence the electricity market prices as well. The capacity expansion algorithm can be used to evaluate investment decisions in a mix of generation technologies, including renewable technologies such as wind and big solar generation.

In this paper, a case study illustrates the capabilities of the Genersys capacity expansion algorithm. Genersys successfully models the complexities and uncertainties in an integrated way facilitating better generation expansion planning.

Keywords: Electricity market modelling, Generation expansion, Agent-based simulation, Investment, Decision Support System (DSS) 


\section{INTRODUCTION}

In an environment of increasing complexity, with the introduction of new electricity generation technologies, climate change, carbon pricing, and fluctuating fuel prices, electricity generation expansion planners need tools which facilitate the integration and synthesis of these and other variables in order to support decision making for investment.

With demand for electricity increasing in many countries due to population growth, economic growth, rising living standards, and climate change impact (Bianco et al 2009, Miller et al 2007) there is an ongoing need to expand electricity generation capacity to meet this demand and to maintain energy security. Building new generation capacity is an expensive and long term venture. Electricity generator companies must make decisions about what type of technology and size of generating plant to invest in, when to build, and where to build in order to maximise their return on investment. These decisions are influenced by many variables.

The cost of building a new generator plant is dependent on what type of technology it uses and when it is built. Improvements in technology and economies of scale due to increased up-take of some technologies can reduce costs over time (Hearps \& McConnell 2011). Operating costs of generator plants vary depending on technology and fuel type used. Fuel costs vary over time and place and are influenced by local and international politics and events. The introduction of carbon pricing schemes brings another variable into play. Mandatory renewable energy targets and other government policy can also influence costs.

In deciding whether or not to invest in a new generator plant consideration must also be given to anticipated revenue. This is partly dependent on the capacity of the technology used - can it generate (and be paid for) electricity most of the time or is its capacity limited by weather conditions (e.g. wind) or other factors? Is it a base, intermediate or peak load supplier? Revenue is also dependent on electricity price which varies over time according to demand and supply. The act of building a new generator plant in itself influences supply and therefore the electricity price which determines revenue. Demand is influenced by climate, population growth, and economic growth.

With so many variables and uncertainties it can be difficult for companies to make investment decisions about when and where to invest in new electricity generation capacity and what technology to employ. Over time, different methodologies, models, and tools have been developed in an attempt to assist decision makers considering electricity generation expansion. Each has it own strengths and weaknesses.

Reviews of existing electricity market models generally and generation expansion models in particular have been conducted by numerous people including Connolly et al (2009), Foley et al (2010), Weidlich \& Veit (2008), and Zhou et al (2009). Gaps identified include non-inclusion of an independent system operator, not modelling transmission losses, and non-consideration of environmental issues such as greenhouse gas emissions. Most generation expansion models do not model the impact of climate on electricity markets, and some include only a limited set of technologies. Models vary in both time resolutions (hourly, daily, yearly, etc.) and time-frame covered (1 year, 50 years, unlimited, etc.). Some model electricity prices, carbon prices, and/or generation expansion exogenously rather than endogenously and many do not model the impact of government policy. The models also vary in their ease of use.

This paper describes a new generation expansion model integrated with the agent-based application Genersys. Genersys is a scenario-based, decision support tool that simulates Australia's electricity and gas markets. Genersys' generation expansion model addresses some of the gaps identified with existing generation expansion models. Section 2 of the paper describes Genersys in general; Section 3 explains how Genersys models generation expansion; Section 4 describes in detail Genersys' dynamic generation expansion algorithm; Section 5 contains a case study that illustrates the capabilities of the Genersys dynamic generation expansion algorithm; and Section 6 draws conclusions about the use of Genersys to model generation expansion.

\section{GENERSYS}

Genersys is an agent-based software application for simulating electricity and gas markets. It was developed by CSIRO's Energy Transformed Flagship in collaboration with Core Energy Group and AGL Energy using object-oriented technology and the Java programming language (Genersys, 2011). It has easy to use interfaces for defining scenarios, viewing simulations, and viewing simulation results. It integrates multiple models which define the characteristics and behaviours of the different aspects of the energy market.

In the Genersys electricity model, generator companies own generating plants which are located in regions. Generator plants consist of one or more generating units, each using a particular type of technology to 
generate electricity, and each with its own characteristics including capacity, fuel type, efficiency, and availability. The electricity network is modelled as an inter-connected regional grid with transmission interconnectors linking the regions.

Genersys simulates the electricity market at 30 minute intervals over a desired period, for example, 30 years. The regional electricity demand model within Genersys is based on a method developed by Thatcher (Thatcher 2007). Demand data sets are constructed at 30 minute intervals. The data is consistent with climate change scenarios and takes into account economic growth factors. Generator companies submit bids for each generating unit to supply electricity into the market to match this demand. An independent market operator balances demand and supply, using submitted bids to determine the amount of electricity to be dispatched by each generating unit, together with the spot price for each region.

A set of bidding models are available for use in Genersys, including dynamic and adaptive bidding. Costbased bids are frequently used for scenario development as they are based on the economic costs of supply for each generating unit. Cost-based bids factor in carbon price, fuel prices, operation and maintenance costs, generation efficiency, and minimum generation restrictions. Optionally, cost-based bidding can factor in fixed operating and maintenance costs, capital costs, and generation capacity factors.

In addition to modelling fossil-fuel based technologies, Genersys has special models for renewable generation including wind, hydro, and solar. These models are linked to climate variables such as temperature, wind speed, water inflows, and solar radiation. Mandatory renewable energy targets are also modelled.

Genersys models greenhouse gas emissions and includes a carbon pricing mechanism. A carbon price may be defined exogenously using an explicit fixed carbon price trajectory. Alternatively an emissions target may be defined and the carbon price is endogenously determined in response to the level of emissions output by the system.

The Genersys electricity model is integrated with the Genersys gas model. The gas model simulates gas supply and demand from gas fields, through gas pipelines and to delivery points. Delivery points include gasbased electricity generators.

To run a Genersys simulation, a scenario is defined via an easy to use graphical user interface. While the simulation is running, its progress can be viewed via an interactive map and many dynamic graphs. Once the simulation has completed its run, the simulation results can be viewed via a report interface. Results include electricity production, spot prices, greenhouse gas emissions, blackouts, revenue, among others.

Alternative scenarios may be explored within Genersys. For example, two different scenarios may be defined which use different carbon price trajectories. After the two simulations have been run, results can be compared to explore the impact of carbon pricing.

\section{GENERSYS GENERATION EXPANSION}

Within a Genersys simulation, as demand for electricity grows over a multi-year period, the existing generation capacity within the simulated market system may no longer be sufficient to meet this growing demand. This usually results in power outages ('blackouts'). The amount of electric energy not delivered to customers due to outages is known as 'unserved energy' (USE). The USE is defined as the amount of energy that cannot be supplied because there is not enough generation to meet the demand. USE is usually calculated as a percentage in relation to the total energy supplied for a period of one year. In order to meet growing demand and avoid blackouts and an unacceptable level of USE, Genersys can simulate generation expansion.

Within Genersys, generation expansion can be modelled in two ways. The first method relies on explicitly defining the new generation capacity when the scenario is defined. When a Genersys scenario is created, all existing generating units are specified including where they are located, what technology they use, and when they start to operate. New generating units may be planned to begin operating in the future. In this way, during the simulation, objects representing the specified new generating units are created at the appropriate time and start to participate in the bidding and supply process, thereby expanding generation capacity. Additionally, generating units can be decommissioned at specified times in the future. Multiple scenarios can be created with different options for generation expansion and the simulation results compared. This 'trialand-error' method can be used to determine the 'best' generation expansion option.

The second method that Genersys provides for generation capacity expansion is a dynamic method. During a simulation, the system responds to USE and pricing signals and automatically adds new generating units, if 
economically viable, as the simulation progresses. Genersys decides where, when, and what type of technology to invest in based on regularly performed economic cost-benefit analyses.

Because the generation expansion algorithm is fully integrated within Genersys it has the capacity to model and assess complex investment scenarios. It enables exploration of different climate and demand patterns, bidding behaviours of generators, carbon and fuel prices, technology investment costs, renewable generation, and all the other components modelled by this simulation tool. The next Section of this paper describes the Genersys dynamic generation expansion algorithm.

\section{GENERSYS DYNAMIC GENERATION EXPANSION ALGORITHM}

When a Genersys scenario is created, investment conditions are defined for each region. Each region has a portfolio of generation technologies that it can invest in. The portfolio may include scheduled (baseload, intermediate or peak load) and non-scheduled (for example wind and large solar) technologies. The size (MW) of potential new generating units is also defined for each technology. Additionally, each region has an upper limit (MW) for investment in each technology type.

During the simulation, an investment check for a region can be triggered in two ways. The first trigger is based on the unserved energy (USE) level. When the USE value for the last simulated year exceeds a given threshold the algorithm will start to evaluate generation technologies for investment in. The second trigger is time based. An explicit repetitive scheduling of the algorithm is defined for the region. For example, it may be set to start three months into the simulation and to occur every six months.

Figure 1 shows a flowchart of the Genersys generation expansion algorithm. It is invoked for each time-step within a simulation.

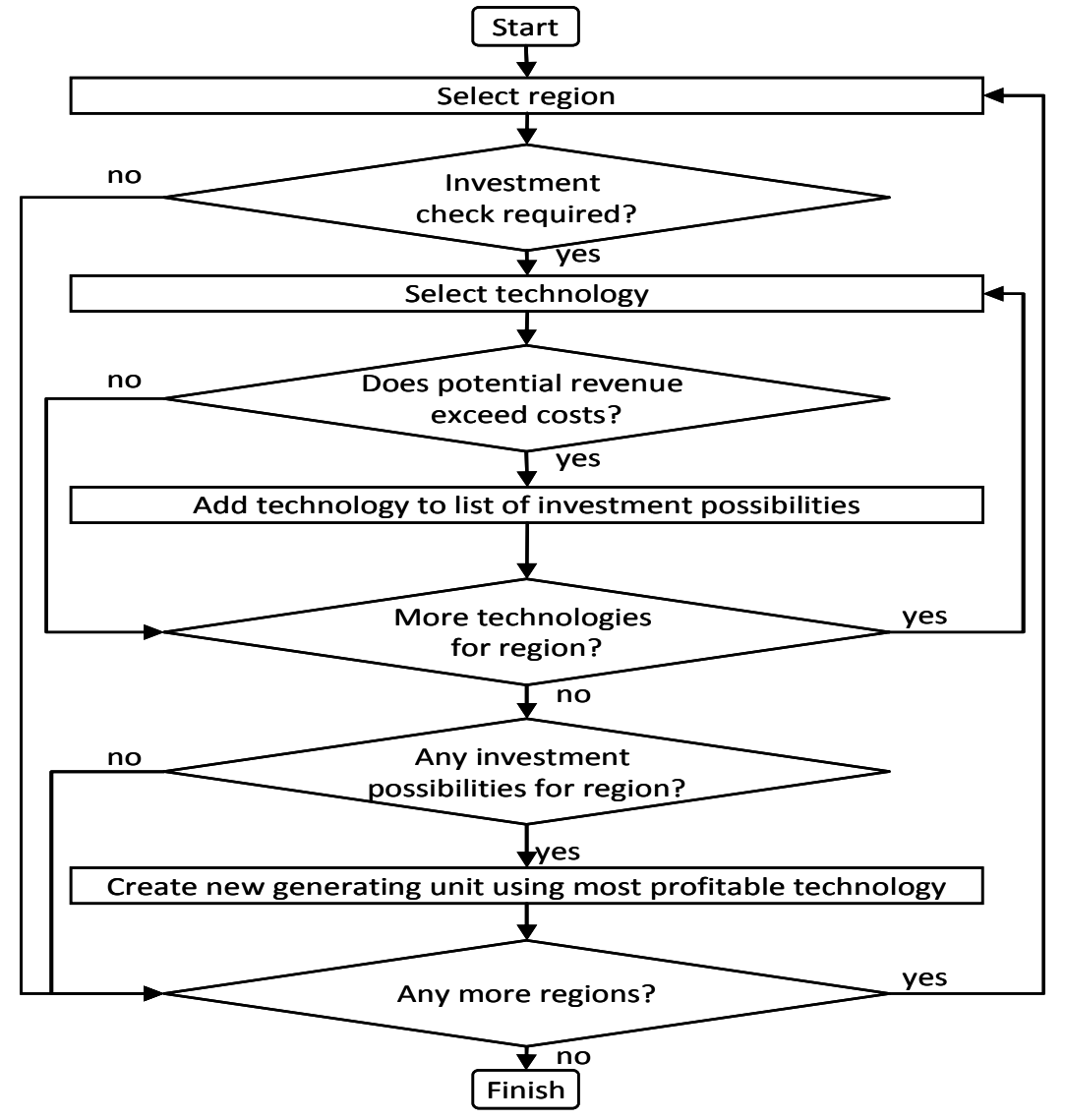

Figure 1: Flowchart of the Genersys dynamic generation expansion algorithm

Once an investment check has been triggered, each technology specified within the region's investment portfolio is assessed to determine whether it would be economically viable to invest in that technology at the current simulation point in time. To determine economic viability, the revenue that could have been achieved by the technology during the year preceding the current simulation time is calculated. Revenue is a function of the electricity prices over the past year, the capacity of the technology, its efficiency, and the type of load 
it is intended to serve (base, intermediate or peak). The costs for the period, both variable (fuel, carbon, variable operating and maintenance) and fixed (capital costs, fixed operating and maintenance), are subtracted from this revenue. The technology that achieves the greatest return is chosen for investment and one new generating unit is created for the region. The new generating unit then participates in the market, supplying bids to the market operator and producing electricity according to dispatch targets. It will have immediate impact on the balance between demand and supply and ultimately influence electricity market prices as well.

\section{CASE STUDY}

A case study based on the Australian National Electricity Market (NEM) illustrates the capabilities of the Genersys dynamic generation expansion algorithm. The NEM is a wholesale electricity market and it operates one of the world's longest interconnected power systems, which includes the States of Queensland, New South Wales, Victoria, Tasmania, South Australia, and the Australian Capital Territory.

A 20 year simulation period was used, starting from 2010. Generation capacity of 49,000 MW is situated within five regions/States across 238 generating units. Generation technologies include conventional black coal, conventional brown coal, hydro, open cycle gas turbine (OCGT), combined cycle gas turbine (CCGT), wind, and biomass. Generation capacity is based on AEMO's 2010 Electricity Statement of Opportunities (AEMO 2010).

In the simulation, electricity demand is calculated based on a linear regression model described by Thatcher (2007). It uses climate data derived from the CSIRO Mk3.0 coupled atmosphere-ocean General Circulation Model (GCM) after being dynamically downscaled by the CSIRO Conformal Cubic Atmospheric Model (McGregor 2005) as part of a future climate projection under the Intergovernmental Panel on Climate Change (IPCC) Special Report on Emissions Scenarios (SRES) A2 scenario (IPCC 2000). Economic growth rates are based on AEMO's 2010 Electricity Statement of Opportunities (AEMO 2010).

The simulation includes a mandatory renewable energy target (MRET). The values used were obtained from the Office of the Renewable Energy Regulator website (ORER 2010).

Each region has a portfolio of generation technologies in which it is able to invest, selected from wind, geothermal, OCGT, CCGT, CCGT with carbon capture and storage (CCS), Integrated Gasification Combined Cycle (IGCC) brown coal, IGCC black coal, IGCC brown coal with CCS, IGCC black coal with CCS, supercritical brown coal, supercritical black coal, supercritical brown coal with CCS, and supercritical black coal with CCS. Each investment generation technology has a capital cost profile associated with it and fuel prices are defined for each region. The discount rate used in the simulation is $9 \%$. An investment check is conducted every 6 months for each region/State and also if the USE is greater than $0.002 \%$ (a reliability value regulated for the NEM (AEMO 2010)).

For the base case a Carbon price trend was used that aims for a $25 \%$ emissions reduction on 2000 levels.

The simulation results (see Figure 2 and Figure 3) show that only three technology types were invested in over the 20 year simulation period - CCGT, OCGT, and wind. Wind and OCGT are not invested in until 2018 and 2019 respectively.

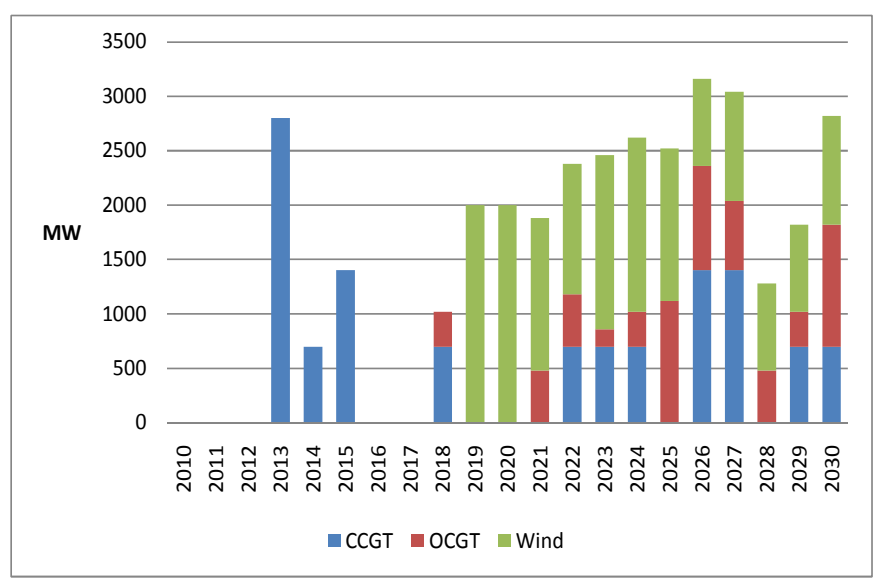

Figure 2: Generation capacity expansion 2010-2030 by year: with carbon price

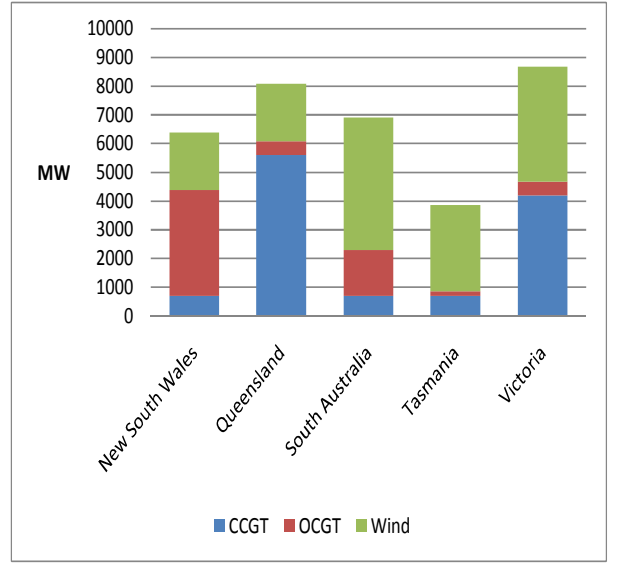

Figure 3: Generation capacity expansion 2010-2030 by region: with carbon price 
A second simulation was run using an alternate scenario that had no carbon price but was otherwise identical to the first. As Figure 4 and Figure 5 show, although the same three technologies were invested in (OCGT, CCGT, and wind), there was much less wind (6,800 MW compared with 15,600 MW) and CCGT (700 MW compared with 11,900 MW), and much more OCGT (15,360 MW compared with 6,400 MW).

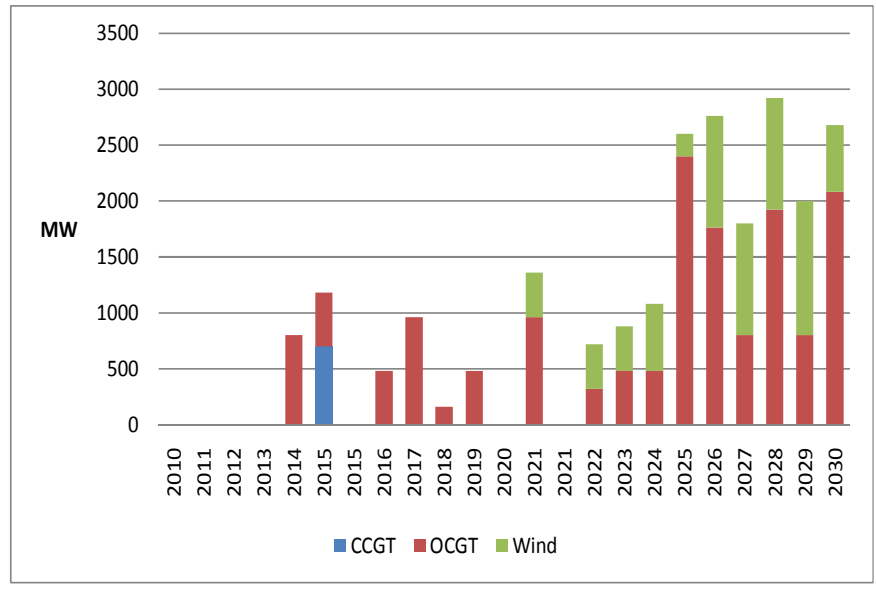

Figure 4: Generation capacity expansion 2010-2030 by year by year: no carbon price

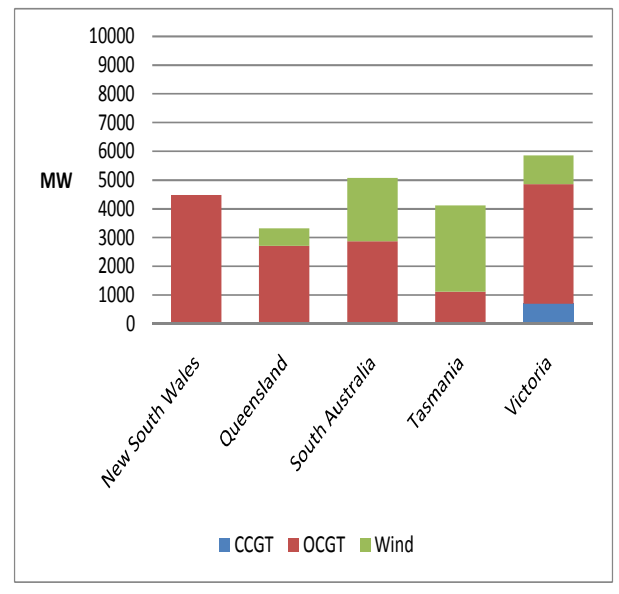

Figure 5: Generation capacity expansion 2010-2030 by region: no carbon price

The two case study simulations show how Genersys can be used to explore different scenarios for generation expansion. In this case the impact of different carbon price trajectories was explored. Further simulations could be run to analyse the impact of the many other variables within the electricity market on generation expansion decisions. This could include variations in capital cost trajectories, technology types and characteristics, MRET targets, fuel costs, climate, and economic growth.

Genersys can be used by energy planners, generator companies, market operators, researchers, and energy consultants to explore multiple scenarios and assist decision making for investment in generation expansion.

\section{CONCLUSION}

Decision making for investment in electricity generation expansion requires analysis and synthesis of a wide range of complex and often uncertain information. A scenario-based decision support tool that models these complexities and uncertainties in an integrated way would facilitate generation expansion planning.

The Genersys generation expansion model integrated with the agent-based Genersys electricity and gas simulation tool provides an easy to use scenario-based tool that through simulation can assist generation expansion planners in deciding when, where, and in what technologies to invest. Genersys' agent-based approach is well-suited to modelling the complexity of electricity markets and the interactions between the various participants and observing emerging market behaviour. Genersys addresses many of the gaps in current generation expansion models. It models electricity prices and generation expansion endogenously and has the capacity to model carbon prices as well. Climate is modeled and it impacts both demand and supply (wind and solar generation). Its 30 minute time resolution means that demand and supply variations can be more accurately modelled and its long time-frame (> 30 years) means that it can model long-term expansion planning. It includes an independent market operator, models transmission losses, greenhouse gas emissions, and some government policy, enables dynamic bidding, and can invest in a wide portfolio of generation technologies including renewables.

The case study showed an example of how Genersys was used to determine a cost effective way of investing in electricity generation expansion in the Australian National Electricity Market given a particular carbon price trajectory, technology cost trajectories, and fuel cost trajectories. The simulation output showed when investment occurred in each region and which technologies were chosen as the most profitable at that point in time. A second scenario showed how not having a carbon price impacted generation expansion.

Genersys could be used by generation expansion planners to analyse a wide range of scenarios. Further case studies could explore the impact of different fuel prices, carbon prices, technology costs, government policy and climate on investment in generation expansion. 
Genersys could be further enhanced by better modelling of distributed generation and the inclusion of storage and demand side participation. Furthermore, currently only one generating unit can be added per region at each investment check period and the capacity of that new generating unit is fixed during scenario definition. Having the capacity to dynamically determine how many units to add and of what capacity would improve Genersys' ability to respond to unserved energy signals.

\section{ACKNOWLEDGEMENTS}

Genersys was developed by CSIRO Energy Transformed Flagship in collaboration with Core Energy Group and AGL Energy. The authors would particularly like to thank Tennyson Wickham of Core Energy Group and Per da Silva previously of CSIRO for their contribution to the Genersys dynamic generation expansion algorithm. The authors would also like to thank Chi-hsiang Wang and Daniel East for their helpful comments on this paper.

\section{REFERENCES}

Australian Energy Market Operator (AEMO) (2010). Electricity Statement of Opportunities. Accessed 6/5/2011 http://www.aemo.com.au/planning/esoo2010.html

Bianco, V., Manca, O., and Nardini, S. (2009). Electricity consumption forecasting in Italy using linear regression models, Energy. 34, 1413-1421.

Connolly, D., Lund, H., Mathiesen, B.V., and Leahy, M. (2009). A review of computer tools for analyzing the integration of renewable energy into energy systems, Applied Energy. 87(2010), 1059-1082.

Foley, A.M., O’Gallachoir, B.P., Hur, J., Baldick, R., and McKeogh, E.J. (2010). A strategic review of electricity systems models, Energy. 35(2010), 4522-4530.

Genersys (2011), CSIRO. Genersys energy modelling and simulation, Accessed 6/5/2011 http://www.genersysenergymodelling.com.au/

Hearps, P. and McConnell, D. (2011). Renewable Energy Technology Cost Review, Melbourne Energy Institute Technical Paper Series.

Intergovernmental Panel on Climate Change (IPCC) (2000). IPCC Special Report Emissions Scenarios.

McGregor, J.L. (2005). C-CAM: Geometric aspects and dynamical formulation. CSIRO Marine and Atmospheric Research Technical Report 70. Accessed 6/5/2011 http://www.cmar.csiro.au/eprint/open/mcgregor_2005a.pdf

Miller, N.L., Hayhoe, K., Jin, J., and Auffhammer, M. (2007). Climate, extreme heat, and electricity demand in California, Journal of Applied Meteorology and Climatology. 47, 1834-1844.

Office of the Renewable Energy Regulator (ORER) (2010). Accessed 6/5/2011 http://www.orer.gov.au/lretsres-updates/index.html\#powerstations

Thatcher, M.J. (2007). Modelling changes to electricity demand load duration curves as a consequence of predicted climate change for Australia, Energy. 32, 1647-1659.

Weidlich, A. and Veit, D. (2008). A critical survey of agent-based wholesale electricity market models, Energy Economics. 30(2008), 1728-1759.

Zhou, Z., Chan, W.K., and Chow, J.H. (2009), 'Agent-based simulation of electricity markets: a survey of tools', Artificial Intelligence Review, 28, 305-342. 